\title{
Social Experiences of Children with Autism at Pathways Autism Trust during the COVID - 19 Lockdown
}

\author{
Pamela Victoria Chakombera $^{1^{*}}$, Philip Mtandwa ${ }^{2}$ \\ ${ }^{I}$ Great Zimbabwe University MSc Counselling Psychology graduate, Zimbabwe \\ ${ }^{2}$ Lecturer: Midlands State University: Faculty of Social Sciences, Zimbabwe
}

*Corresponding Author: Pamela Victoria Chakombera, Great Zimbabwe University, MSc Psychology Masters graduate, Zimbabwe.Email: vchakombera92@gmail.com

\begin{abstract}
The research looked into the social experiences of children with autism at Pathways Autism Trust. The major issue of concern was inquiring on the social experiences of children living with autism at the institution. The aim was exploring how COVID-19 affected the activities of daily living for these children as they are routine oriented and disorientation would come with unintended consequences or outcomes. Therefore the research was governed by the interpretivist research paradigm, utilised a qualitative research and experiences explored through the phenomenological research design. The population was acquired through the convenience sampling as the participants were drawn centrally the Pathways Autism Trust. The researchers found significant negative effects on the lives of the participants. The major finding was on disruption of activities of daily living and the plausibility of post COVID-19 negative effects. Therefore it was concluded that, the COVID-19 era affected significantly on the overall life experiences and as such there is need to develop safety nets for the post COVID-19 era.
\end{abstract}

Keywords: Social, experiences, children, autism, COVID-19, lockdown, caregivers.

ABBREVIATIONS: COVID-19 - Coronavirus disease of 2019, MERS - Middle East Respiratory Syndrome, SARS - Severe Acute Respiratory Syndrome, WHO - World Health Organisation.

\section{INTRODUCTION}

The year 2020 saw nation's worldwide lockdown to avoid the spread of COVID - 19. Children living with autism have experienced a sudden change in their routine day to day activities. They are resistant to change and the lockdowns have presented psycho- social problems, such problems include distress, anxiety, meltdowns, non-contact with relevant professions, among others. Children on the Autistic Spectrum are likely to come out of the lockdown with unintended unfavourable psychologicalbehavioural manifestations. Thus the aim of the study will be to explore the ways in which children at Pathways Autism Trust are psychologically experiencing the lockdown and the potential areas of concern in these experiences during and post-Covid era.

The history of epidemics and pandemics can be traced back to 430 B.C.E 'Plague of Athens' in the Greek city of Athens, 300 B.C.E malaria in Ancient Rome, 165 C.E plague of the Antonines [1] and recently the worldwide 20th century COVID-19. Government decrees declared restrictive measures in order to prevent the wider spread of COVID-19 and these include lockdowns [2]. Confinements have a long history worldwide too, these were and are still adopted to deal with the spread of diseases, and such pandemics that have utilised confinements include the Black Death, great plague, sweating sickness, typhoid fever, Middle East Respiratory Syndrome (MERS), Severe Acute Respiratory Syndrome (SARS) and recently COVID -19. The past decade has seen Africa experience its own share of confinements as a way to curb spread of disease for example the Ebola epidemic in western and central Africa [3]. Recently, most countries worldwide have been subjected to the COVID - 19 lockdown, most countries in Africa have been compliant to regulations set by World Health Organisation (2020) to curb the spread of the disease. Zimbabwe has not been exempt to the lockdown either.

Lockdowns come with routine change for individuals, this adjustment presents a distress to general populaces and even worse to people living with autism and their families. The rapid changes in the 
environment of operations produces psycho-social consequences that hinder activities of daily living in at least $60 \%$ in people living with Autistic Spectrum Disorders [4]. The sudden changes alters the experiences of reality, and simultaneously triggers adaptation changes to both people and environments for persons with Autism. Confinements might be complex for children with autism who react disproportionately to changes in their daily routine. In critical situations such as pandemics there might changes that occur in terms of the mental health of children with autism.

Most families with children living with autism rely on professionals such as therapists and support personnel who provide sensory input and emotional support to the children. The lockdown left parents with children living with autism in a lurch as they are not able to source out these services [5]. Obsessions, repetitive behaviour and routines are a way of life and a source of pleasure for children living with autism. Children living with autism like and need routine, routine is an element that calms and organizes their day to day lives [6]. Children living with autism are resistant to change and the period might see them exhibiting strong resistance to the lockdown change.

Some countries took necessary measures to deal with the repercussions of the lockdown on children with autism. France president Emmanuel Macron on World Autism Day adjusted Frances confinement rules for people with autism [7]. The nation allowed them to go out and to visit places they habitually visited prior to the lockdown. News24 South Africa, reported that the South Africa's autism community made a plea to the South African government for the urgent lockdown concessions to restore routine support services [8]. The Zimbabwean government exempted certain health-related facilities but sadly autism centres in the country were not included, thus Pathways Autism Trust closed during the lockdown. Pathways Autism Trust is a non-governmental organisation that was founded by parents of autistic children. The organisation provides autism-specific educational and therapeutic services for children living with autism at the centre.

Children with autism find it difficult to cope with change as they insist on sameness, they normally find it problematic to adjust to change of routine. Findings in Portugal suggested that children with autism that did not have scheduled routines or generally maintain routines reported higher levels of anxiety than those that maintained scheduled routines [9].In a qualitative study reports indicated that children with autism would visibly get frustrated when a parent tried to transition them from one activity to the other [10]. The restricted access to regular programming interrupts various domains of an autistic individual's life these include mental health and physical health [11]. It is from the above that one may note that change has negative implications on children living with autism.

Researchfindings suggest that children with autism experience sensory sensitivity particularly tactile defensiveness [12]. Children with autism may not be comfortable wearing masks over their faces, they could possibly find them irritating. This in turn might come with negative implications such as stigma and discrimination as people would not want to interact with them fear of being exposed to the COVID -19 virus. Findings in Italy offer contradictory evidence suggest that there were no changes on taste/smell sensitivity in children with autism during the COVID-19 pandemic [13]. Thus it is inconclusive whether there are changes or no changes in general sensitivity.

Educational systems worldwide closed due to the COVID- 19 pandemic, this interfered with children with autism school routine activities. Different initiatives were introduced in different countries to ensure that children do not lag behind. These serve the purpose of educating children outside the school maintaining their literacy and numeracy. Research findings in Zimbabwe uncovered that parents with children living with autism drew material from the internet to help their children [14]. Educational manuals have been produced by experts to help caregivers and families of children living with autism to educate them in their homesteads.

Schools are regarded as an essential service for autism communities, they are regarded as critical for they provide special education, social interaction and most importantly regulate their behaviour. Research findings in Turkey found that a majority of children with autism stopped receiving special education during the COVID-19 pandemic [15].Research findings suggest that individuals with autism who received indirect school support during the lockdown exhibited less intense behaviour problems as compared to those that did not [16]. The closure of school facilities both increases and decreases stress in individuals with autism [17].

People with autism struggle with change and have difficulties interacting with other people and the pandemic could likely amplify these challenges [18]. They further are highly likely to have increased 
levels of distress due to the amplification of the difficulties. High levels of depression were noted in people living with autism during the COVID-19 lockdown [19] and these could be attributed to the presenting change. Research findings suggest that children with autism deteriorated in terms of social, developmental and behavioral domains during the pandemic [20].

The global occurrence of autism was questioned years ago, it was taken to exist only in western countries. However, the last decade has seen knowledge and prevalence of autism being documented as being on the rise in different parts of the world [21]. Knowledge and awareness of autism is generally low in Africa, particularly sub-Saharan Africa [22]. Not many studies have been carried out in Zimbabwe, thus there is little knowledge related to the area particularly confinements and children living with autism. Due to the exceptional nature of the changes to family life occurring during the COVID-19 pandemic and the dearth of research on this topic, it was necessary to implement a qualitative study to identify the range of experiences of autistic children under confinements in Harare province, Zimbabwe.

\section{Statement Of The Problem}

The year 2020 saw nation's worldwide lockdown to avoid the spread of COVID - 19. Children living with autism have experienced a sudden change in their routine day to day activities. They are resistant to change and the lockdowns have presented psycho-social problems, such problems include distress, aggression, meltdowns, and non-contact with relevant professions, among others. Children on the Autistic Spectrum are likely to come out of the lockdown with unintended unfavourable psychological-behavioural manifestations. Thus the aim of the study will be to explore the ways in which children at Pathways Autism Trust are psychologically experiencing the lockdown and the potential areas of concern in these experiences during and post-Covid era.

\section{RESEARCH OBJECTIVE}

To explore the social experiences of children living with autism during the COVID - 19 lockdown.

\section{Materials ANd Methods}

The study was governed by the interpretivist paradigm as its research philosophy. Under this philosophical underpinning, the research then adopted a qualitative research approach because of its integrity in simplifying and managing data without destroying the data's complexity and context. The purpose of the research was to explore the psychological experiences of children living with autism during the COVID-19 pandemic. This was achieved by learning from the children's care givers in the way they viewed the pandemic's effects on the children, the meanings they put on the pandemic and how they interpreted their experiences. An interpretivist phenomenological design was utilized to understand the structure of the children's lived experiences during the COVID - 19 lockdown.

\section{TARGET POPULATION}

The target population of this study was 9 home-based care givers of children with autism at Pathways Autism Trust. The participants to this research were children who had at least been diagnosed with Autism Spectrum Disorder for more than a year.

\section{Sample And Sampling Procedure}

The study sample was drawn from Pathways Autism Trust. The researchers utilised a sample of at least four (4) caregivers, with at least 2 females and 2 males based on availability to the organisation and referral from the organisation. Through convenience sampling as participants were from an organization and highly accessible, the total number of participants of this study was determined by data saturation, a state with which no new information was drawn from the participants.

\section{DAta Collection Instruments}

A semi-structured interview was used, the interview guide is a research instrument which has the capacity to not only to record practices and standards but also achieve them, challenge them as well as reinforce them. The researchers recorded the respondents whilst they gave their accounts, this was done using electronic recording devices. The researchers then coded the data gathered and a thematic based approach was used utilizing the thematic analysis six stages to come up with findings on the social experiences of the participants 


\section{Findings OF The STUdy}

The findings from the study demonstrate that social experiences of children under the lock down were vast. There was a strong realization of the diversity in experiences based on care giver to child relation, child to environment relation amongst other social experiences. Therefore, from the study, the research managed to capture social experiences such as self-isolation and alienation, prolonged lockdown social effect, social sensing and withdrawal, alienated mental health, abrupt social change and social anxiety, online learning and autistic functioning and lastly social compromise. These are as presented below:

\subsection{Self-Isolation and Alienation}

The introduction of lockdowns played down social development of children in the autistic spectrum. The findings from the study demonstrated that most of these children's social behaviour receded and some even lost touch with their self. Care givers reported that they could not identify what was now wrong and how it had become as such, but attributions pointed towards a change in the general norms of operation. However others believed that the lock down improved relations with other siblings.

Participant 1

"............he now spends more time with his sibling who is usually away as he is enrolled at a boarding school, I think this would help him integrate into the society as he is being exposed to non-autistic individuals........."

Participant 3

".......................it so happens that I as the father was a victim of Covid 19, now imagine a situation where my son is used to being around me and all of a sudden, no school and I am not around him, he couldn't understand, and at times he would try and come into my room and deterring him made him miserable ..................."

Participant 2

“......................there hasn't been much to talk about in terms of social improvement except that he gets to spend more time with his siblings and my mother

The findings from the study may be demonstrating that the lack of usual attention to the child may have long lasting implications. The findings may also be suggesting that slight changes in affection and physical contact can affect the child's trust level. These findings may be suggesting that emotional development and social relations in children living with autism are bi-polar in nature and have a devastating implication.

\subsection{The Prolonged Lockdown Effect}

As lockdown has impacted negatively on their routine at the Pathways Autism Trust, these children are now becoming acculturated to the new environment and developing a liking for the new environment which in turn might affect their re-integration into the centre. The findings from the study demonstrated that some of the parents had managed to cope with the lock-down and amongst them, most were from environments where there was only one child and their attention was central to them. Those with more children found it difficult to handle and have the child disassociate with other children as it interfered with their routine behaviour and obsessive nature of their life.

Participant 1

“...................the lockdown came with its own challenges, my child's routine was disrupted and he cannot ask because he is incapacitated to do so, thus it has been hard on him to take it in.................."

Participant 4

".....................in as much as it is difficult for them to relate and interact with them, it has been critical for my child to relate with family and be familiar with them, at times when I leave him or if one of his close family members leaves for example when his father tested positive for Covid 19 and had to self-isolate,....................he showed signs of separation anxiety and was very happy to see our return which I feel is a mile stone in his emotional development. 
Participant 3

".........................ever since the lockdown was instituted, I can see that my son does not understand why he is no longer going to school, what happened to his school mates, those are the people he relates to even on social events such as birthdays. So his world has been minimized and we don't know how this will turn out on him in the long run.... ,'

Participant 2

“ I am worried that my child will return to the centre lagging from lack of support from the centre, but also he has developed some skills that are basic to us who see it and minimal behaviors

The findings may also be demonstrating that the lockdown did not bring much in terms of social development but rather it only improved social relations. The findings from the study may be demonstrating that the major negative impact was disruption of the daily routine of the children. The findings from the study may have also suggested that familiarization with other family members also took a huge tore on the children especially if there were new members to the family. The findings may also suggest that, the abrupt change caused a lot of confusion in the children because they do not know or understand what is going on.

\subsection{Social Sensitivity and Withdrawal}

The concept of social distancing and social isolation proved not to work well with children in autistic spectrum. The challenges associated did not account that these children do not understand what it means to be removed from the general social circle they are used to. Thus during this pandemic, sleep and management of routine behaviour was also noted as a contributory factor on how their withdrawal emanated. The changes in the care giver's schedule, with the work from home concept meant social deprivation during hours which the child saw the parent, these children did not understand that availability of the parent at home did not imply that they are there to play and attend to them as they are used to.

Participant 4

"..................... find my child as the sensitive type, but what I have grown to like is that his siblings are just there for him, they make sure he is always in a safe space. He is a person with attention deficit hyperactivity challenges which means he needs that kind of special attention..........."

Participant 3

during the period I was in self-isolation, I felt terrible, my son was could not be with him, he could only see me as socially withdrawing which made him withdraw too emotional, socially and psychologically.... ,."

The findings from the study may have indicated that children with autism are sensitive in nature. These findings may have suggested that they need a small circle of people for them to thrive. The findings from the study may be illustrating that autism can be coupled with other challenges such as ADHD, which on their own pose a huge threat on their wellbeing. These findings demonstrated that children living with autism's needs are sensitive and need proper moulding in order to reach desired outcomes and goals in socialization.

\subsection{Indifferent Mental Health Care}

The findings from the study demonstrated that the majority of children that utilized Pathways Autism Trust had access to speech, social and other services providers who are specialized in therapies. The abrupt disruption in access left more care givers clueless on what they have to do and how they are supposed to support their children, these children on the other hand have been left with a void, people that are usually in their daily social routine have been removed and this has impacted significantly on the general social wellbeing of the children and caregivers. 
Participant 3

"........................although it has been a challenge to access medication, we have tried by all means to support our child with all the needs he demands ",

Participant 2

"........................sometimes we use medical systems, but however over the course of the lockdown there has been significant changes to this, I usually sit with him and his favourite toys trying to calm his nerves, but pretty challenging for a person like me who is not well trained in that domain..................."

Evidence from the study may have pointed towards abrupt closure of health services related to autistic care. The findings may be illustrating that some of the children heavily rely on medication which may prove inaccessible. The findings from the study suggest that it is important for consideration of lockdown effect to account for the lives of people with special needs before imposition.

\title{
8.5. Abrupt Social Change and Social Anxiety
}

The changes that have happened around the world has taken a lot from us, however children with autism have limited resources to understand these changes. Changes in their environment have a significant effect in how they process social emotions, the majority of care givers demonstrated that these changes coming in simultaneously disrupted their limited scope of social interactions. These challenges have affected children differently with the majority of caregivers reporting highly explosive behaviour in their children. The combination of such behaviors and the chronic fatigue that caregivers reported resembled a disenabling environment for both individuals.

Participant 4

".........................the challenges that Covid 19 brought on my child are irrefutable bad, however I have also grown to appreciate the experiences that he has shared with family. The positive experience with family has helped me see my child building a resilient character one that may enable him to reintegrate with other people besides family. ,

Participant 3

"...................the routines that our child goes through, he does not understand them but rather complies because we reinforce the behaviors, so when instructions come in simultaneously and rapidly, they disorient the child... ,"

Participant 2

\begin{abstract}
"......................my son has been used to me taking him with me and putting him in school, but these changes in the environment has negatively affected him. He does not comprehend why I no longer take him with me, and so our relation is likely to reside..., he doesn't understand but to him the routine is crucial.
\end{abstract}

The findings from the study proposed that some of the caregivers have resorted to conditioning their children to comply with the demands of the lockdown. The findings may also be suggesting that social reintegration may be a challenge for it will heavily rely on memory and access for the child to remember his friends from before at the centre. The findings may also be suggesting that most of these children remain confused and this has triggered behaviors that present as new to the ill equipped caregivers to deal with.

\subsection{Online Learning and Autistic Functioning}

The concept of learning to the majority of children speaks to a shift of physical utilization of paper and pen to online resources. This function was undermined for children with mild to high functioning autism. Caregivers of children with high functioning autism have constantly reported anxious behaviour and irritability they are not able to use computers or machines, the introduction of these aspects to them resembled toys and not resources. The case was rather different for these suffering from moderate autistic traits, these children could relate although their concentration span remain low. 
The resolve from the caregivers was that although these amenities sort to improve their cognitive functioning, these children would develop attachments to the gadgets and utilized them as they saw fit, further drowning them into their own social worlds.

Participant 4

It's unfortunate that these may not be relatable or relevant to the children, it's only us as the parents who can get online support but it's always a challenge. What we have made sure is to stay connected with other people and specialists who can help us cope with the spectrum condition. ,"

The findings from the study may be suggesting that online learning and other online and computer based services are not conducive for children living with autism. These findings may be suggesting that these children's learning has been totally stopped as compared to others without special needs. Such findings demonstrated on the long term negative consequences of such a gap in treatment.

\subsection{Social Compromise}

Caregivers reported that in most instances it is important to understand the mental age of the child against their chronological age. The evidence suggested that most of the children with autism have challenges in comprehending the world, as such expecting them to operate at their frustration level only affects them further. Their way of life is filled with compliments and the role needs a caregiver to give in more hours of their lives, which at times presents itself as stressful. Thus, the little communicative gestures that these children make need to be recognized and appreciated because the child significance towards their social growth and development especially if they are more challenged on the communicative spectrum.

Participant 4

"...................interacting more closely with the family and taking his medication has made him more maturely behaved, and I strongly believe the positive interaction with family has improved him although he cannot express it because he cannot speak......................."

Participant 3

“....................sometimes our child can exhibit extreme behaviors, at one time he pushed his grandmother against the wall, so it's up to us as parents to try and make sure we reinforce positive change, this behaviour is not rather a consequence of the lockdown but inherent to him "

Participant 2

".....................staying with him at home is difficult, sometimes he throws tantrums and hits against furniture, the home environment is not adequate and it does not suit him well compared to the Pathways environment, so we usually resort to medicines that compose him..... ,

Evidence gathered from the study may be suggesting that as parents, there is great need for social and emotional compromise when dealing with children living with autism. The findings may also be suggesting that caregivers need to give the children the much needed attention and patience to make them better. The findings from the study proposed that some of the children would throw tantrums and exhibit physical outbursts but such need special care to manage for them to improve. These findings demonstrate that living with autism is a special way of life that needs externalized understanding.

\section{Discussion Of RESults}

Social reduction of experiences, social alienation and social anxiety were amongst the major challenges that affected children living with autism during the lockdown. Reports indicated that children with autism would visibly get frustrated when a parent tried to transition them from one activity to the other [23]. These notions pointed out that such changes affect the manner in which interaction occurs within the realms of the children and the people around them. Research evidence supported this by asserting that restricted access to regular programming interrupts various domains of 
an autistic individual's life these include mental health and physical health, as such these attributes of life tend to topple balance in the life of children with autism [24]. These sensory stimulations that come in with great magnitude affects these children's social sensing capabilities and fostering withdrawal from unscheduled or unfamiliar activities, environments and faces.

Social comprehension of events also stood out as a domineering factor in the life of children living with autism. The evidence from the suggested that some of the children chronological and mental age are not synchronised and as such the communicative, restrictions, obsessive behaviours and respective nature in routine symbolises their level of intellectual functioning. The findings from the study resorted to the notion that, caregivers and those around the child will need an elevated compassion, sympathy, patience and subtle traits that improve the quality of the relationship with the child. Children living with autism are not feeling and sensing in terms of mind theory, therefore it is upon the caregiver to experience his/ her world and incorporate their feelings, perceptions and perspectives in order to understand their world.

A drastic change in the social environment amongst some of the participants was coupled with hostile behaviour and tantrums and affected the manner in which these children function. Failure to take precautionary measures like priming and giving warnings could possibly lead to problem behaviours such as tantrums, crying and aggressive behaviours [25]. Their general function proved to be anchored around familiarity and routine behaviour, thus due to limitations in social behaviour or verbal communicative behaviour affects their behavioural manifestation, which present itself as selfinduced or social induced violent behaviour.

\section{CONCLUSION}

Based on the findings and literature obtained from the study, the researchers concluded that there were significant alterations in activates of daily living for both care givers and children living with autism. The findings also demonstrated that, the change was rapid and forced which made life difficult to adjust for the mentioned categories of participants. The findings also brought to light that autism is a lifestyle and not a disorder, therefore, the incoming COVID- 19 lockdowns disrupted this life style and as such post COVID-19 consequences are to be anticipated. The re-adjustment to normalcy after COVID-19 will also bring with it a phase for another drastic change, thus making the lifestyle more and more disrupted and with unpredictable outcomes. Therefore based on all these evaluations, researchers recommends that the government and other stakeholders take into cognoscente these areas of concern and work with them accordingly.

Conclusions drawn have also highlighted that communication, interactions and relations between children with autism and their parents are critical for the children's health and mental wellbeing. More so, the community needs to understand what autism is, this enlightenment would help them appreciate autism. As the saying goes 'children are products of their communities' community members are likely to develop a sense of responsibility and participate in helping children and families with autistic children. More over the post COVID-19 era will require counsellors to come up with interventions that are specific and particular in assisting autistic children when dealing with confinements. The research will probably allow counsellors to develop research based interventions informed by evidence gathered from a population with the characteristics that are representative to children with autism.

\section{REFERENCES}

[1] Tognotti, E. (2013). Lessons from the history of quarantine, from plague to influenza A. Emerging infectious diseases, 19(2), 254.

[2] Devi, S. (2020). Travel restrictions hampering COVID-19 response. The Lancet, 395(10233), 1331-1332.

[3] Burd, E. M. (2015). Ebola virus: a clear and present danger. Journal of clinical microbiology, 53(1), 4-8.

[4] Nisticò, V., Gambini, O., Faggioli, R., \&Demartini, B. (2020). The psychological impact of COVID-19 among a sample of Italian individuals with High Functioning Autism Spectrum Disorder.

[5] Simon, S. (2020, April 4). Lockdown leaves parents of autistic children in the lurch Lockdown leaves parents of autistic children in the lurch. Retrieved from https:/www.newindianexpress.com/cities/ thiruvananthapuram/2020/apr/04/lockdown-leaves-parents-of-autistic-children-in-the-lurch-2125394.html

[6] Richler, J., Bishop, S. L., Kleinke, J. R., \& Lord, C. (2007). Restricted and repetitive behaviors in young children with autism spectrum disorders. Journal of autism and developmental disorders, 37(1), 73-85.

[7] Web24 News (2020, April 2) Emmanuel Macron announces a containment arrangement for autistic people. Retrieved from https://www.web24.news/u/2020/04/emmanuel-macron-announces-a-containmentarrangement-for-autistic-people.html 
[8] Broughton, T. (2020, April 26) Covid-19: Autism community pleads for relaxed lockdown rules. News24. Retrieved from https://www.news24.com/SouthAfrica/News/covid-19-autism-community-pleads-forrelaxed-lockdown-rules-20200426

[9] Amorim, R., Catarino, S., Miragaia, P., Ferreras, C., Viana, V., \&Guardiano, M. (2020). The impact of COVID-19 on children with autism spectrum disorder.

[10] Parenteau, C. I., Bent, S., Hendren, R. L., Hossain, B., Chen, Y., Widjaja, F., \&Breard, M. (2020). 51.1 A QUALITATIVE ANALYSIS: THE EXPERIENCE OF PARENTS OF CHILDREN WITH AUTISM SPECTRUM DISORDER DURING THE COVID-19 PANDEMIC. Journal of the American Academy of Child and Adolescent Psychiatry, 59(10), S251.

[11] Bandini, L. G., Curtin, C., Phillips, S., Anderson, S. E., Maslin, M., \& Must, A. (2017). Changes in food selectivity in children with autism spectrum disorder. Journal of autism and developmental disorders, 47(2), 439-446.

[12] Güçlü, B., Tanidir, C., Mukaddes, N. M., \&Ünal, F. (2007). Tactile sensitivity of normal and autistic children. Somatosensory \& motor research, 24(1-2), 21-33.

[13] Di Renzo, M., Di Castelbianco, F. B., Vanadia, E., Petrillo, M., D'Errico, S., Racinaro, L., \& Rea, M. (2020). Parent-Reported Behavioural Changes in Children with Autism Spectrum Disorder during the COVID-19 Lockdown in Italy. Continuity in Education, 1(1), 117-125. DOI: http://doi.org/10.5334/cie.20

[14] Majoko, T., \&Dudu, A. (2020). Parents' strategies for home educating their children with Autism Spectrum Disorder during the COVID-19 period in Zimbabwe. International Journal of Developmental Disabilities, 1-5.

[15] Mutluer, T., Doenyas, C., \&Genc, H. A. (2020). Behavioral Implications of the Covid-19 Process for Autism Spectrum Disorder, and Individuals' Comprehension of and Reactions to the Pandemic Conditions. Frontiers in psychiatry, 11.

[16] Parenteau, C. I., Bent, S., Hendren, R. L., Hossain, B., Chen, Y., Widjaja, F., \&Breard, M. (2020). 51.1 A QUALITATIVE ANALYSIS: THE EXPERIENCE OF PARENTS OF CHILDREN WITH AUTISM SPECTRUM DISORDER DURING THE COVID-19 PANDEMIC. Journal of the American Academy of Child and Adolescent Psychiatry, 59(10), S251.

[17] Turk, M. A., Landes, S. D., Formica, M. K., \& Goss, K. D. (2020). Intellectual and developmental disability and COVID-19 case-fatality trends: TriNetX analysis. Disability and Health Journal, 13(3), 100942.

[18] Patel, A. Badiani, F. Nielsen, S Assi, V Unadkat, B Patel, K Courtney, L. Hallas, COVID-19 and Autism: Uncertainty, Distress and Feeling Forgotten, Public Health in Practice, https://doi.org/10.1016/j.puhip. 2020.100034.

[19] Baribeau DA, Vigod S, Pullenayegum E, Kerns CM, Mirenda P, Smith IM, Vaillancourt T, Volden J, Waddell C, Zwaigenbaum L, et al. Repetitive behavior severity as an early indicator of risk for elevated anxiety symptoms in autism spectrum disorder. J Am Acad Child Adolesc Psychiatry. 2020;59(7): 890-9 e893.

[20] TokatlyLatzer, I., Leitner, Y., \&Karnieli-Miller, O. Core experiences of parents of children with autism during the COVID-19 pandemic lockdown. Autism, 1362361320984317.

[21] Bakare, M. O., \&Munir, K. M. (2011). Autism spectrum disorders (ASD) in Africa: a perspective. African journal of psychiatry, 14(3), 208-210.

[22] Bakare, M. O., \&Munir, K. M. (2011). Autism spectrum disorders (ASD) in Africa: a perspective. African journal of psychiatry, 14(3), 208-210.

[23] Parenteau, C. I., Bent, S., Hendren, R. L., Hossain, B., Chen, Y., Widjaja, F., \&Breard, M. (2020). 51.1 A QUALITATIVE ANALYSIS: THE EXPERIENCE OF PARENTS OF CHILDREN WITH AUTISM SPECTRUM DISORDER DURING THE COVID-19 PANDEMIC. Journal of the American Academy of Child and Adolescent Psychiatry, 59(10), S251.

[24] Bandini, L. G., Curtin, C., Phillips, S., Anderson, S. E., Maslin, M., \& Must, A. (2017). Changes in food selectivity in children with autism spectrum disorder. Journal of autism and developmental disorders, 47(2), 439-446.

[25] Schreibman, L., Whalen, C., \&Stahmer, A. C. (2000). The use of video priming to reduce disruptive transition behavior in children with autism. Journal of positive behavior interventions, 2(1), 3-11. 


\section{AUTHORS' BIOGRAPHY}

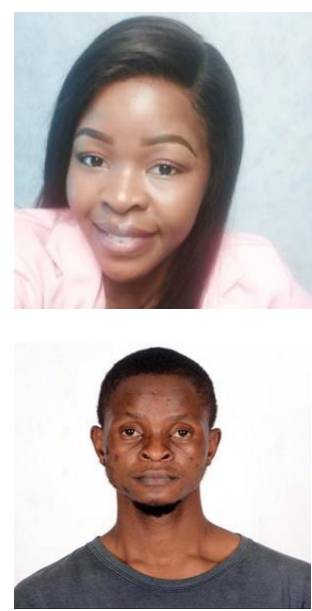

Pamela Victoria Chakombera, is a Human Resource Practioner and a woman of letters who is devoted to the generation of knowledge in different areas of psychology. Her research interests are child psychology, gender issues and sexism, counselling psychology, political psychology and industrial psychology.

Phillip Mutandwa, is an academia who has made research at local, regional and international level. He has contributed towards knowledge production in several areas including his role as a reviewer in other international journals. His keen areas are on pragmatic aspects of psychology and psychological functions in society through evidence based practices and simple psychology for communities.

Citation: Pamela Victoria Chakombera, Philip Mtandwa. "Social Experiences of Children with Autism at Pathways Autism Trust during the COVID - 19 Lockdown" International Journal of Humanities Social Sciences and Education (IJHSSE), vol 8, no. 9, 2021, pp. 141-150. doi: https://doi.org/10.20431/2349. 0381.0809014 .

Copyright: () 2021 Authors. This is an open-access article distributed under the terms of the Creative Commons Attribution License, which permits unrestricted use, distribution, and reproduction in any medium, provided the original author and source are credited. 